\title{
Alterações neuroendócrinas em pacientes com traumatismo cranioencefálico
}

\author{
Leonardo de Moura Sousa Júnior1, luri Santana Neville1, Djalma Felipe da Silva \\ Menendez', Malebranche Berardo Carneiro da Cunha Neto², Rafael Loch Batista ${ }^{2}$ \\ Eberval Gadelha Figueiredo ${ }^{3}$, Almir Ferreira de Andrade ${ }^{4}$, Manoel Jacobsen Teixeira ${ }^{5}$
}

Departamento de Neurocirurgia do Hospital das Clínicas da Faculdade de Medicina da Universidade de São Paulo (HC-FMUSP), São Paulo, Brasil.

\section{RESUMO}

O traumatismo cranioencefálico (TCE) corresponde a uma das principais causas de morte em adultos jovens. Alguns pacientes com TCE podem ser vítimas de várias alterações hormonais decorrentes do trauma. Algumas são facilmente reconhecíveis, como diabetes insipidus, enquanto outras podem passar despercebidas inicialmente, como a deficiência do hormônio do crescimento (GH). As alterações neuroendócrinas após a ocorrência de trauma podem cursar com deficiências da hipófise anterior, da posterior ou de ambas, acometer apenas um eixo hormonal ou vários e, ainda, ser transitórias ou permanentes. A grande maioria dos pacientes que apresentam disfunção neuroendócrina foi vítima de traumas considerados moderados ou graves pela escala de Glasgow. No entanto, a maioria dos estudos não evidenciou relação entre a gravidade da lesão e a ocorrência de alteração hipofisária pósTCE. As deficiências hipofisárias devem ser tratadas precocemente, uma vez que déficits hormonais hipofisários dificultam a recuperação dos pacientes traumatizados, constituindo-se em fator de pior prognóstico. Apesar da frequência com que ocorrem os TCE, existem poucos estudos a respeito das complicações neuroendócrinas decorrentes.

\section{PALAVRAS-CHAVE}

Traumatismos craniocerebrais, hipófise, hipopituitarismo.

\section{ABSTRACT}

Neuroendocrine disorders in traumatic brain injury patients

Traumatic brain injury (TBI) is one of the main causes of death in young adults. Some cases of TBI could lead to the development of easily recognizable diabetes insipidus. In other cases it can lead to alterations in the endocrine axis that are more difficult to notice at the beginning and that can occur together with other hormone deficiencies. The majority of patients with neuroendrocrine dysfunction were TBI victims with a Glasgow Coma Scale scores of 3-13 (moderate and severe trauma). However, previous studies did not show correlationships between the severity of injury and pan-hypopituitarism after TBI. The pituitary disorders have to be treated as soon as possible because it makes the recovery of TBI patients more difficult and lead to a worse prognosis. Despite the number of studies about TBI, actually there are only a few studies concerning the secondary neuroendocrine complications.

\section{KEYWORDS}

Craniocerebral trauma, pituitary gland, hypopituitarism.

1 Médico-residente de Neurocirurgia do Hospital das Clínicas da Faculdade de Medicina da Universidade de São Paulo (HC-FMUSP), São Paulo, SP, Brasil.

2 Médico neuroendocrinologista do Departamento de Neurocirurgia do Instituto de Psiquiatria (IPq) da FMUSP, São Paulo, SP, Brasil.

3 Supervisor da Divisão de Neurocirurgia do HC-FMUSP, São Paulo, SP, Brasil.

4 Diretor técnico do Pronto-Socorro de Neurocirurgia do HC-FMUSP, São Paulo, SP, Brasil.

5 Professor titular do Departamento de Neurocirurgia do HC-FMUSP, São Paulo, SP, Brasil. 


\section{Introdução}

O traumatismo cranioencefálico (TCE) corresponde a uma das principais causas de morte em adultos jovens. É caracterizado por qualquer agressão que acarrete lesão anatômica ou comprometimento funcional do crânio e/ ou estruturas internas. ${ }^{1-3} \mathrm{O}$ TCE ocorre mais frequentemente em indivíduos entre 15 e 24 anos, em crianças com menos de 5 anos; homens apresentam risco duas vezes maior do que mulheres.

A glândula hipófise, por sua localização anatômica, pode ser facilmente atingida em casos de TCE, especialmente nos casos mais graves. Essa glândula possui aproximadamente $1 \mathrm{~cm}$ de diâmetro e pesa cerca de $1 \mathrm{~g}$. Situa-se na sela turca, na base do cérebro, e conecta-se ao hipotálamo pelo pedículo ou haste hipofisária. Pode ser dividida em adeno-hipófise, ou hipófise anterior, e neuro-hipófise, ou hipófise posterior. A anterior deriva-se de uma invaginação embrionária do epitélio faríngeo, enquanto a posterior origina-se de uma protuberância do tecido neural hipotalâmico. ${ }^{4}$

- A hipófise anterior possui diferentes tipos de células, que são especializadas em sintetizar hormônios específicos:

- Somatrotofos $\rightarrow$ hormônio do crescimento humano $(\mathrm{GH})$

- Corticocotrofos $\rightarrow$ adrenocorticotropina (ACTH)

- Tireotrofos $\rightarrow$ hormônio tireoestimulante (TSH)

- $\quad$ Gonadotrofos $\rightarrow$ hormônio luteinizante (LH) e hormônio folículo-estimulante (FSH)

- $\quad$ Lactrotofos $\rightarrow$ prolactina (PRL)

Cerca de 30\% a 40\% das células da hipófise anterior são constituídas de somatrotofos, enquanto $20 \%$, de corticotrofos. Os outros tipos de células constituem, cada um, cerca de 3\% a 5\% do total. A hipófise anterior possui grande quantidade de sinusoides entre as células glandulares. $\mathrm{O}$ sangue que penetra nesses sinusoides passa inicialmente na parte inferior do hipotálamo e mantém seu trajeto para a adeno-hipófise por meio de pequenos vasos porta-hipotalâmico-hipofisários. Por meio desse sistema, hormônios hipotalâmicos influenciam a secreção dos hormônios hipofisários, seja estimulando ou inibindo sua liberação.

- Hormônio de liberação de tireotropina (TRH): promove liberação do hormônio tireoestimulante.

- Hormônio de liberação de corticotropina (CRH): promove liberação de corticotropina.

- Hormônio de liberação do hormônio do crescimento (GHRH): promove liberação do hormônio do crescimento.

- Hormônio de inibição do hormônio do crescimento (GHIH), também denominado somatostatina: inibe a liberação do hormônio do crescimento.

- Hormônio de liberação das gonadrotopinas $(\mathrm{GnRH})$ : promove a liberação dos hormônios gonadrotópicos - LH e FSH.

- Hormônio de inibição de prolactina (PIH): inibe a secreção da prolactina.

A localização anatômica da glândula hipófise colabora para a sua predisposição ao trauma. Entre os mecanismos de lesão hipofisária, podem ser citados: compressão da glândula e/ou edema com compressão dos núcleos hipotalâmicos, fratura craniana com lesão concomitante das estruturas adjacentes, hemorragias, aumento da pressão intracraniana, hipóxia ou lesões diretas ao hipotálamo, haste hipofisária ou hipófise. Assim sendo, dependendo da agressão, os vasos do sistema porta-hipotalâmico-hipofisário podem ser afetados, causando isquemia da adeno-hipófise. Também pode ser observada hiperprolactinemia, já que as lesões traumáticas podem acometer a contrarregulação desse hormônio, produzida pela secreção hipotalâmica de dopamina. ${ }^{5}$

Apesar da frequência com que ocorrem os TCE, existem poucos estudos a respeito das complicações neuroendócrinas secundárias.

Assim sendo, procuramos revisar dados publicados a respeito da ocorrência de alterações neuroendócrinas em pacientes vítimas de TCE.

\section{Metodologia}

Este artigo constituiu-se de revisão de literatura sobre distúrbios neuroendócrinos em pacientes com TCE. Foram revisados estudos disponíveis sobre esse tema publicados até janeiro de 2012.

\section{Resultados}

Foram revisadas 22 publicações relacionadas à temática estudada, as quais identificaram a presença de disfunção neuroendócrina em pacientes vítimas de TCE. No entanto, verificamos grande variação entre os trabalhos sobre a prevalência dessas alterações.

Alguns estudos encontraram prevalência de até $100 \%$ de alterações do eixo gonadal, enquanto outros evidenciaram apenas $42 \%$ de alteração neuroendócrina.

Estudos de autópsia mostraram que até $86 \%$ dos pacientes que morrem agudamente por TCE apresentam 
evidências de lesões hemorrágicas no hipotálamo, na hipófise ou na haste hipofisária.

No entanto, a metodologia para se identificarem as disfunções hormonais pós-TCE foram muito discrepantes entre os estudos, o que pode explicar, ao menos em parte, as diferentes prevalências encontradas.

\section{Discussão}

O TCE corresponde a uma das principais causas de morte em adultos jovens. Sabe-se, no entanto, que a real incidência de traumatismos é subestimada, tanto pela não assistência médica aos casos leves quanto pela evolução desfavorável dos traumatismos severos antes mesmo do socorro médico.

Pacientes com TCE podem ser estratificados em níveis de gravidade segundo alguns critérios. TCE leve: pacientes admitidos com nível de consciência de 13 a 15 pontos na ECG. A incidência do TCE leve gira em torno de 300.000 casos novos por ano nos Estados Unidos, sendo ainda considerado um número subestimado, e tais pacientes permanecem internados de $52 \%$ a $72 \%$. TCE grave: pacientes admitidos com nível de consciência de 3 a 9 pontos na ECG após 6 horas do TCE. ${ }^{1-3}$

Boa parte dos pacientes com TCE moderado/grave, após medidas iniciais, pode ser vítima de fatores agravantes que inicialmente podem passar despercebidas como relato de deficiências hormonais em variadas combinações.

Tanto deficiências neuro-hipofisárias quanto adeno-hipofisárias podem estar presentes, e o tempo decorrente entre o TCE e suas repercussões clínicas pode ser bem variado. Algumas deficiências hormonais permanecem anos sem serem reconhecidas. $\mathrm{O}$ profissional de saúde deve estar atento a tal condição, pois não raramente nem o paciente se recorda mais do evento traumático prévio, e cuidadosa anamnese pode recuperar essa importante informação.

O hipopituitarismo pode ser subclínico, sendo apenas diagnosticado após exames hormonais, ou apresentar sintomas como amenorreia, infertilidade, disfunções eréteis, astenia, alterações psíquicas, intolerância ao frio (devida ao hipotireoidismo central), hipotensão (devida ao hipocortisolismo central) e galactorreia (devida à hiperprolactinemia). ${ }^{6,7}$

O estado de coma dificulta o diagnóstico pós-traumático, e muitos médicos associam as deficiências pós-traumáticas a síndromes pós-concussionais. No entanto, deficiências dos hormônios adrenais, bem como dos hormônios tireoidianos, contribuem para o estado comatoso.

Em geral, as primeiras deficiências hormonais a surgirem nos casos de hipopituitarismo pós-TCE são as do
GH e das gonadotropinas, provavelmente decorrentes da posição anatômica das células somatotrópicas e gonadotrópicas na hipófise e sua relação com a vascularização, que pode ser facilmente afetada logo após o trauma.

Segundo Benvenga et al., ${ }^{6}$ o eixo gonadotrófico é o mais acometido pelo trauma: quase $100 \%$ dos casos apresentaram deficiências de FSH e LH. Deficiências de TSH e ACTH estavam presentes em quase $50 \%$ dos casos. As deficiências de GH (refletidas na diminuição de IGF-1) e PRL estavam presentes em aproximadamente $23 \%$ dos casos.

A criança possui maior propensão ao TCE comparada aos adultos, em razão de suas características anatômicas: maior relação cabeça-corpo e menor espessura do crânio, por exemplo. ${ }^{8}$ Também existem poucos trabalhos a respeito de alterações neuroendócrinas em crianças vítimas de TCE. Niederland et al. ${ }^{9}$ publicaram um estudo com 26 crianças (17 meninos e 9 meninas) vítimas de TCE, submetidas a testes de estímulos hormonais para avaliação de suas funções hipofisárias, por um período de três anos após o evento traumático. O trabalho revelou que $23 / 26$ pacientes não apresentavam sequelas neurológicas, porém $60 \%$ das crianças avaliadas apresentaram alguma deficiência neuroendócrina. ${ }^{8-10}$

Tanriverdi et al. ${ }^{11}$ verificaram que nas dosagens imediatas pós-trauma, em $41,6 \%$ dos pacientes ocorreram deficiência gonadotrófica (LH e FSH), em 20,4\%, deficiência de GH, em $12 \%$ encontram-se níveis elevados de prolactina, em 9,8\%, deficiência de ACTH, em 5,8\%, deficiência de TSH, enquanto $51,9 \%$ apresentaram a síndrome do T3 baixo (T4 livre normal e níveis baixos de T3 livre). Descreveu-se uma correlação positiva entre os níveis de testosterona livre e os valores da escala de coma de Glasgow, enquanto os níveis de prolactina foram negativamente relacionados à mesma escala. Numa avaliação 12 meses após o trauma cranioencefálico, os mesmos autores relatam que $32,7 \%$ dos pacientes apresentaram deficiência somatotrófica (níveis séricos de hormônio de crescimento menores ou iguais a 10 $\mathrm{mg} / \mathrm{l}$ ), testados pela administração de GHRH (fator liberador de hormônio de crescimento) e GHRP-6 (peptídeo liberador de hormônio de crescimento). A deficiência corticotrófica foi encontrada em $25 \%$ dos pacientes com níveis basais de cortisol abaixo de $7 \mathrm{mg} / \mathrm{dl}$. A deficiência gonadotrófica foi observada em $7,7 \%$ dos pacientes e foi associada à moderada hiperprolactinemia. Sob estímulo de TRH (fator hipotalâmico liberador de TSH), 5,8\% dos pacientes não responderam ao teste, sendo considerados deficientes em TSH. Após um ano de evolução, os pacientes avaliados por Tanriverdi et $a l .{ }^{11}$ não apresentaram alterações significativas nos eixos corticotrófico e somatotrófico comparados aos dados obtidos pós-trauma, e o eixo gonadotrófico mostrou recuperação. 
Tanriverdi et al. ${ }^{11}$ mostram, em estudo prospectivo avaliando as funções hipofisárias analisadas num período $<12$ horas pós-trauma cranioencefálico e após 12 meses, que a glândula hipofisária responde de muitas formas após um traumatismo cranioencefálico. Numa fase aguda, níveis altos ou baixos de GH basal podem ser observados. Altas concentrações de GH com níveis basais diminuídos de IGF-1 são descritos em pacientes críticos (resistência ao GH). Os dados obtidos neste trabalho mostram que os níveis de GH estão elevados no momento agudo do trauma, comparados com os níveis dosados 12 meses após o trauma, quando o nível de IGF-1 na fase aguda está significativamente mais baixo, comparado com os níveis de IGF-1 após 12 meses do trauma (compatível com resistência periférica ao $\mathrm{GH}$ ).

A maioria dos pacientes que apresentam deficiências hormonais foi vítima de traumas graves, caracterizados pela escala de coma de Glasgow entre 3-13 (traumas graves ou moderados). No entanto, os trabalhos não mostram relação entre a gravidade da lesão e o pan-hipopituitarismo pós-TCE. Tanriverdi et al. ${ }^{11}$ não encontraram diferenças significativas quando avaliaram as funções de diferentes setores endócrinos, tais como função tireoidiana, suprarrenal, gonadal, bem como o crescimento, por meio do eixo GH/IGF-I nos pacientes com lesão cerebral grave, moderada e leve. Contudo, nos indivíduos com lesões cerebrais graves, os níveis de testosterona livre se mostraram menores, comparados aos que sofreram lesões intermediárias e leves. Adicionalmente, Tanriverdi et al. ${ }^{11}$ não mostraram diferenças significativas entre os níveis de IGF-1 dos pacientes com deficiência somatotrófica, comparados àqueles normais. A deficiência de ACTH foi encontrada em $19 \%$ dos casos, representando o segundo eixo com maior deficiência - muito provavelmente pela baixa sensibilidade do teste de ACTH usado para o diagnóstico de insuficiência adrenal.

As elevações do cortisol sérico, com aumento da secreção de ACTH no momento agudo pós-trauma, estão relacionadas com a gravidade da lesão. $\mathrm{O}$ nível de cortisol na fase aguda foi significativamente maior comparado às dosagens um ano após o trauma. Nos pacientes gravemente doentes os níveis de T3 e T4 livre estão diminuídos. Numa série de estudos tem sido descrita a síndrome do T3 baixo. No trabalho realizado por Tanriverdi et al. ${ }^{11}$ há insuficiência do eixo tireotrófico em $51,9 \%$ dos pacientes na fase aguda e em 5,7\% dos pacientes após 12 meses do trauma.

Outros estudos prospectivos sugerem que alterações funcionais que se apresentam logo após o TCE são transitórias, enquanto aquelas que se apresentam mais tardiamente são permanentes. No entanto, não se deve fazer uma avaliação definitiva quanto aos déficits hormonais num período inferior a um ano do TCE. ${ }^{12-14}$
Em relação à gravidade do trauma e à disfunção hipofisária como sua consequência, existem algumas controvérsias. Benvenga et al. ${ }^{6}$ relataram que $93 \%$ dos pacientes com hipopituitarismo pós-TCE ficaram em coma ou inconscientes logo após o trauma. Kelly et al. ${ }^{15}$ identificaram uma pontuação na escala de coma de Glasgow menor que 10 e presença de edema cerebral na tomografia como indicadores de risco para o desenvolvimento de hipopituitarismo após TCE. No entanto, Agha et al..$^{12-14}$ não observaram relação entre a gravidade do trauma e uma consequente disfunção hormonal.

$\mathrm{O}$ hipogonadismo secundário à deficiência de gonadotropinas é comum na fase aguda do TCE. Num estudo 12 dias após o trauma, Agha et al. ${ }^{12}$ mostraram prevalência de $80 \%$ de hipogonadismo. Em longo prazo, a prevalência varia consideravelmente, de $2 \%$ a $32 \%$. A avaliação do eixo hipofisário-gonadal de rotina nos sobreviventes de TCE se faz necessária para o diagnóstico de deficiências hormonais. Entre essas deficiências, o hipogonadismo é reconhecido hoje como frequente complicação secundária ao TCE, contribuindo para a alta morbidade observada nesses pacientes. Hohl et al. ${ }^{16}$ analisando 30 pacientes, sendo 22 homens, com intervalo médio da avaliação laboratorial entre o TCE e o os exames laboratoriais, encontraram $10 \%$ dos pacientes (66,7\% homens) com níveis de FSH abaixo do normal e 3,3\% deles com nível baixo de LH. No grupo masculino, 9,1\% apresentavam níveis de testosterona abaixo do valor de referência. Nesse trabalho foi observada associação entre os níveis baixos de testosterona e valores menores de LH. Todos os pacientes do estudo apresentaram TCE grave e, ao se analisarem os escores de ECG com as dosagens alteradas de FSH, LH e testosterona, concluiu-se não haver associação significativa entre eles para essa população. Isso sugere que pacientes com lesões mais graves, portanto com pior prognóstico, não desenvolveram tardiamente mais alterações dos hormônios estudados em relação aos pacientes com lesões menos graves. Ao contrário deste estudo, Klose et al. ${ }^{17}$ evidenciaram risco aumentado de hipogonadismo pós-traumático em pacientes vítimas de TCE grave.

A falta de GH provoca, em adultos, diversos sinais e sintomas, como aumento da gordura corporal, obesidade abdominal, dislipidemia, resistência insulínica, hipertensão, aumento dos fatores trombóticos e da mortalidade cardiovascular, diminuição da massa óssea e da força muscular, bem como intolerância ao exercício físico. Ainda pode ser encontrada redução da energia física e mental, da memória, da capacidade de concentração, e em alguns pacientes pode ocorrer aumento no transtorno de ansiedade, isolamento social e redução no bem-estar psíquico e físico. ${ }^{18,19}$

Para realização do diagnóstico, são feitos alguns testes provocativos. A resposta inadequada do hormônio a esses testes faria o diagnóstico, sendo recomendados, pelo 
menos, dois testes para confirmar a deficiência de GH. O teste atualmente considerado padrão-ouro é o teste de tolerância à insulina, o qual revela a deficiência de GH grave quando ele se encontra abaixo de $3 \mathrm{mcg} / \mathrm{l}$. Entretanto, esse teste induz o paciente a um estado de hipoglicemia e pode causar convulsões, o que o contraindica em algumas situações, como no caso de doença cardiovascular isquêmica e em pacientes que sofreram TCE. Outro teste a ser efetuado seria o GHRH + arginina, o qual considera deficiência de GH grave um valor inferior a $9 \mathrm{mcg} / \mathrm{l}$. Esse teste mostrou alta sensibilidade e especificidade quando comparado ao ITT, além disso, sua realização é bem mais aceita em condições clínicas como TCE. A dosagem de IGF-1 no plasma também pode ajudar no diagnóstico, sendo fortemente preditivo de deficiência grave quando encontrado em níveis baixos no plasma. ${ }^{20-22}$

Uma vez reconhecida a deficiência de GH, o seu tratamento deverá ser considerado. Foi demonstrado que a melhora clínica com a reposição hormonal ocorre principalmente durante o primeiro ano de tratamento e que a boa resposta terapêutica se assemelha a dos pacientes que apresentam deficiência de GH causada por outras doenças. Com a reposição de $\mathrm{GH}$, é possível se observarem muitos efeitos positivos, como a perda da cintura abdominal e a diminuição da concentração lipídica no plasma, o aumento da massa muscular e da capacidade de realizar exercícios físicos, e a melhora do humor; todos esses itens também contribuem para a recuperação do TCE.

\section{Considerações finais}

Boa parte dos pacientes com TCE moderado/grave, após medidas iniciais, pode ser vítima de fatores agravantes que inicialmente podem passar despercebidas, como relato de deficiências hormonais em variadas combinações.

A grande maioria dos pacientes que apresentam deficiências hormonais foi vítima de traumas graves, caracterizados pela escala de coma de Glasgow entre 3-13 (traumas graves ou moderados). Esses indivíduos apresentam significativa morbidade pós-traumática e deverão ser avaliados com relação a possíveis deficiências hormonais.

As deficiências hipofisárias devem ser tratadas o quanto antes, uma vez que o estado de hipopituitarismo dificulta a recuperação dos pacientes traumatizados, constituindo-se em fator de pior prognóstico. O hipopituitarismo pós-traumático é bem mais frequente do que se pensava, pois lesões cranioencefálicas decorrentes do trauma podem evoluir com deficiências graves dos hormônios hipofisários. Mesmo após décadas do evento traumático, o médico deverá estar atento para as possíveis deficiências decorrentes daquele insulto.

Apesar da frequência com que ocorrem os TCE, existem poucos estudos a respeito das alterações hormonais hipofisárias secundárias ao trauma, o que torna necessária a realização de estudos prospectivos visando a melhor entendimento a respeito da função hipofisária desde as primeiras horas após o TCE e seu comportamento evolutivo, com o objetivo de revelar informações importantes que ajudem no manejo adequado dessas alterações, garantindo menor morbidade e mortalidade relacionadas ao TCE.

\section{Referências}

1. Andrade AF, Marino Jr J, Miura FK, Carvalhaes CC, Tarico MA, Lázaro RS, et al. Diagnóstico e conduta do paciente com traumatismo cranioencefálico leve. In: Projeto Diretrizes: Sociedade Brasileira de Neurocirurgia; 2011.

2. Andrade AF, Marino Júnior J, Miura FK, Rodrigues Jr JC. Traumatismo cranioencefálico grave. In: Projeto Diretrizes: Sociedade Brasileira de Neurocirurgia; 2012.

3. Andrade AF, Marino Junior J, Miura FK, Rodrigues Jr JC. Traumatismo cranioencefálico moderado. In: Projeto Diretrizes: Sociedade Brasileira de Neurocirurgia; 2012.

4. Guyton AC. Tratado de fisiologia médica. $9^{\mathrm{a}}$ ed. Rio de Janeiro: Guanabara; 1997.

5. Keleştimur F. Chronic trauma in sports as a cause of hypopituitarism. Pituitary. 2005;8(3-4):259-62.

6. Benvenga S, Campenní A, Ruggeri RM, Trimarchi F. Clinical review 113: Hypopituitarism secondary to head trauma. J Clin Endocrinol Metab. 2000;85(4):1353-61.

7. Lieberman SA, Oberoi AL, Gilkison CR, Masel BE, Urban RJ. Prevalence of neuroendocrine dysfunction in patients recovering from traumatic brain injury. $\mathrm{J}$ Clin Endocrinol Metab. 2001;86(6):2752-6.

8. Cook RS, Schweer L, Shebesta KF, Hartjes K, Falcone RA Jr. Mild traumatic brain injury in children: just another bump on the head? J Trauma Nurs. 2006;13(2):58-65.

9. Niederland T, Makovi H, Gál V, Andréka B, Abrahám CS, Kovács J. Abnormalities of pituitary function after traumatic brain injury in children. J Neurotrauma. 2007;24(1):119-27.

10. Cohan P, Wang C, McArthur DL, Cook SW, Dusick JR, Armin $B$, et al. Acute secondary adrenal insufficiency after traumatic brain injury: a prospective study. Crit Care Med. 2005;33(10):2358-66.

11. Tanriverdi F, Senyurek H, Unluhizarci K, Selcuklu A, Casanueva FF, Kelestimur F. High risk of hypopituitarism after traumatic brain injury: a prospective investigation of anterior pituitary function in the acute phase and 12 months after trauma. J Clin Endocrinol Metab. 2006;91(6):2105-11.

12. Agha A, Thornton E, O'Kelly P, Tormey W, Phillips J, Thompson CJ. Posterior pituitary dysfunction after traumatic brain injury. J Clin Endocrinol Metab. 2004;89(12):5987-92.

13. Agha A, Sherlock M, Phillips J, Tormey W, Thompson CJ. The natural history of post-traumatic neurohypophysial dysfunction. Eur J Endocrinol. 2005;152(3):371-7.

14. Agha A, Thompson CJ. Anterior pituitary dysfunction following traumatic brain injury (TBI). Clin Endocrinol (Oxf). 2006;64(5):481-8. 
15. Kelly DF, Gonzalo IT, Cohan P, Berman N, Swerdloff R, Wang C. Hypopituitarism following traumatic brain injury and aneurysmal subarachnoid hemorrhage: a preliminary report. J Neurosurg. 2000;93(5):743-52.

16. Hohl A, Mazzuco TL, Coral MH, Schwarzbold M, Walz R. Hypogonadism after traumatic brain injury. Arq Bras Endocrinol Metabol. 2009;53(8):908-14.

17. Klose M, Juul A, Poulsgaard L, Kosteljanetz M, Brennum $\mathrm{J}$, Feldt-Rasmussen U. Prevalence and predictive factors of post-traumatic hypopituitarism. Clin Endocrinol (Oxf). 2007;67(2):193-201.

18. Bondanelli M, Ambrosio MR, Zatelli MC, De Marinis L, degli Uberti EC. Hypopituitarism after traumatic brain injury. Eur J Endocrinol. 2005;152(5):679-91.

19. Cukiert A, Libman B. Neuroendocrinologia clínica e cirúrgica. São Paulo: Lemos; 2002.

20. Ghigo E, Aimaretti G, Arvat E, Camanni F. Growth hormonereleasing hormone combined with arginine or growth hormone secretagogues for the diagnosis of growth hormone deficiency in adults. Endocrine. 2001;15(1):29-38.

21. Consensus guidelines for the diagnosis and treatment of adults with growth hormone deficiency: summary statement of the Growth Hormone Research Society Workshop on Adult Growth Hormone Deficiency. J Clin Endocrinol Metab. 1998;83(2):379-81.

22. Hoffman DM, O'Sullivan AJ, Baxter RC, Ho KK. Diagnosis of growth-hormone deficiency in adults. Lancet. 1994;343(8905):1064-8.

\section{Endereço para correspondência}

Leonardo de Moura Sousa Júnior

Rua Padre Machado, 96, Ed. Carlos Eduardo, ap. 52, Bosque da Saúde

04127-000 - São Paulo, SP, Brasil

Telefone: (11) 7347-7319

E-mail: leonardomoura.jr@hotmail.com 